Orsolya Rauzs

Szeged

\title{
Übersetzungsbewertung und Fehlerkorrektur aus der Perspektive von Studierenden
}

DOI: 10.14232/fest.bassola.30

\begin{abstract}
Vorliegender Artikel befasst sich mit Fragen der Bewertung und Fehlerkorrektur von Übersetzungen. Nach einer kurzen theoretischen Einführung ins Thema fokussiere ich mich auf ausgewählte Ergebnisse einer Erhebung, die ich mit Studierenden durchgeführt habe. Die Erhebung liefert Informationen darüber, was Studierende über die von DozentInnen verwendete holistische Bewertungsmethode denken und inwieweit sie fähig sind, Übersetzungen selber zu bewerten und zu korrigieren.
\end{abstract}

\section{Einleitung}

Als Lehrkraft am Institut für Germanistik der Universität Szeged bin ich seit 10 Jahren am Übersetzungsunterricht beteiligt. Ein wichtiger Teil unserer Seminare ist, dass wir DozentInnen die von den Studierenden produzierten Übersetzungen bewerten und benoten, was natürlich auch einen gewissen methodologisch fundierten Umgang mit Fehlern voraussetzt. Was Fehlerklassifikation und Bewertungsmethode angeht, werden in der Fachliteratur verschiedene Typen präsentiert (s. Kapitel 2), von denen man die für die aktuellen Unterrichtszwecke (und für die Lehrkraft) geeignetste auswählen kann. Möchte man aber dabei auch die Studierenden mit in Betracht ziehen, sind schon einige Fragen unumgänglich:

- Welche Bewertungsmethode finden die Studierenden am fairsten und am nützlichsten?

- $\quad$ Können Studierende anhand der jeweiligen Bewertung ihre Fehler korrigieren bzw. aus ihren Fehlern lernen?

- $\quad$ In welchem Maße sind Studierende fähig, Übersetzungen selber zu korrigieren und $\mathrm{zu}$ bewerten? 
Im Rahmen eines Seminars bin ich diesen Themen nachgegangen. Die Studierenden wurden zu den obigen Punkten befragt, bzw. mussten sie verschiedene Aufgaben zum Bewerten und zur Fehlerkorrektur machen. Der vorliegende Artikel fasst die mithilfe der Erhebung gewonnenen Erkenntnisse zusammen.

\section{2. Überblick über Bewertungsmethoden und Fehlerklassifikationen}

\subsection{Bewertungsmethoden}

Nach Hönig (1997 zitiert nach Dróth 2011: 7) können Übersetzungsbewertungen therapeutische oder diagnostische Ziele haben. Die therapeutische Bewertung gliedert sich in den Unterrichtsvorgang, indem man damit den Studierenden eine Rückmeldung geben möchte, worin sie sich noch verbessern sollten, wobei auch die Gründe der Fehler im Fokus stehen. Die diagnostische Bewertung ist eine reine Beurteilung der übersetzerischen Leistung, etwa aus der Perspektive des potenziellen Lesers.

Bewertungen können sich nach einer (Gruppen-)Norm oder nach einem Kriterium richten. Im ersten Fall wird die Übersetzung im Vergleich zu der Leistung einer bestimmten Gruppe (z.B. der Seminargruppe oder der Profi-ÜbersetzerInnen) oder zu einer bestimmten Qualitätsnorm beurteilt. Im zweiten Fall informiert die Bewertung darüber, wie erfolgreich sich die Studierenden das Lernmaterial eines Kurses angeeignet haben oder in welchem Maße ihre Leistung den curricularen Anforderungen entspricht (Dróth 2017: 296-297).

Die Form der Evaluierung kann holistisch oder analytisch (oder gemischt) sein. Holistische Bewertungen bestehen aus globalen Formulierungen, die den allgemeinen Eindruck der BewerterInnen zu bestimmten Übersetzungsaspekten widerspiegeln. Analytisch kann man die Anzahl der verschiedenen Fehler - gewichtet oder ungewichtet - mit der Leistungsqualität in Verbindung setzen (Dróth 2011: 10).

\subsection{Fehlerklassifikationen}

In der Fachliteratur findet man verschiedene Fehlerklassifikationen, die nach diversen Kriterien aufgestellt werden. Die Heterogenität zeigt beispielsweise die folgende Liste von Klaudy (2007: 256-257): 
- $\quad$ Typologie nach den Ursachen der Fehler

- Verständnisfehler / Rezeptionsfehler

- Transferfehler

- Produktionsfehler

- Unachtsamkeitsfehler

- $\quad$ Typologie nach dem Grad der Informationsvermittlung

- im Zieltext steht etwas Anderes als im Ausgangstext

- im Zieltext steht mehr als im Ausgangstext

- im Zieltext steht weniger als im Ausgangstext

- $\quad$ Typologie nach linguistischen Kategorien

- lexikalische Fehler

- morphologische Fehler

- syntaktische Fehler

- usw.

- $\quad$ Typologie nach Fehlerebene

- Fehler auf Wortebene

- Fehler auf Satzebene

- Fehler auf Textebene

- stilistische Fehler

- pragmatische Fehler

Je nach individueller Auffassung oder institutionellen Vorgaben können die einzelnen Fehlerklassen auch gewichtet berücksichtigt werden (s. Klaudy 2007: 257, Kußmaul 2015: 179, Nord 2010: 181-183, Stolze 2013: 385).

Bei Übersetzungen sollten eigentlich nur die Kompetenzen und Leistungen bewertet werden, die beim jeweiligen Auftrag und bei der geforderten Qualitätsstufe ${ }^{1}$ relevant sind. Als fehlerhaft sollten daher nur Elemente gelten, die den Kriterien des Auftrags nicht entsprechen (vgl. Dróth 2011: 8, Nord 2010: 179-181). So bezieht beispielsweise Nord (2010: 180-181) Fehler auf den jeweiligen Übersetzungsauftrag und unterscheidet 3+1 Fehlertypen:

Eine Rohübersetzung ist inhaltlich korrekt, allerdings können in ihr sprachliche und stilistische Fehler vorkommen. Eine Informations-/Arbeitsübersetzung muss auch sprachlich wohlformuliert und stilistisch akzeptabel sein. Druckreif ist ein Text, der sowohl inhaltlich als auch sprachlich und stilistisch angemessen ist und nur noch formatiert werden muss. Wenn auch das erfolgt, ist der Text eine druckfertige Übersetzung (vgl. Koller 2011: 207, Schäffner 2004: 113). 
- $\quad$ pragmatische Übersetzungsfehler: Nichtberücksichtigung der Funktion des Zieltextes und der Kommunikationssituation

- $\quad$ kulturelle Übersetzungsfehler: Nichteinhaltung der Konventionen der Zielkultur

- $\quad$ sprachliche Übersetzungsfehler: Verstöße gegen sprachliche Normen, deren Einhaltung vom Auftraggeber gefordert ist

- $\quad$ sonstige sprachliche Fehler: Verstöße gegen sprachliche Normen, deren Einhaltung vom Auftraggeber nicht gefordert ist (z.B. bei einer Rohübersetzung)

Standardisierte (analytische) Bewertungsmodelle mit Fehlerklassen und Fehlergewichtung wurden ebenfalls entwickelt, z.B. das LISA QA Model², das SAE J2450 Model $^{3}$ oder das neue MQM ${ }^{4}$, vor allem für industrielle Zwecke und Qualitätssicherung. Als Beispiel dient hier die Liste der Fehlertypen bzw. -ebenen von MQM (MQM 2015: 5.2):

- $\quad$ Accuracy (Addition, Improper exact TM match, Mistranslation, Omission, Untranslated)

- Design (Length, Local formatting, Markup, Missing text, Truncation/ text expansion)

- $\quad$ Fluency (Character encoding, Grammatical register, Grammar, Inconsistency, Link/cross-reference, Punctuation, Spelling)

- $\quad$ Locale convention (Address format, Date format, Currency format, Measurement format, Shortcut key, Telephone format)

- $\quad$ Style (Awkward, Company style, Inconsistent style, Third-party style, Unidiomatic)

- $\quad$ Terminology (Inconsistent with termbase, Company terminology, Thirdparty termbase, Inconsistent use of terminology)

- $\quad$ Verity (Culture-specific reference)

Entwickelt von der Localization Industry Standards Association (LISA).

Entwickelt von der Society of Automotive Engineers (SAE).

Multidimensional Quality Metrics, entwickelt vom Deutschen Forschungszentrum für Künstliche Intelligenz GmbH und QTLaunchPad. 
Die didaktische Fehlerklassifizierung und Bewertung unterscheiden sich aber von der Berufspraxis: Im Unterricht „erfolgt die Evaluation von Übungsleistungen stets als Übersetzungskritik in einer statischen Fehleranalyse aufgrund des Vergleichs der Übersetzung mit dem Ausgangstext“ (Stolze 2013: 385). Da Übersetzungsseminare auch die Sprachkompetenzen verbessern bzw. gute Sprachkenntnisse voraussetzen, wird häufig auf sprachliche Fehler - vor allem beim Übersetzen in die Fremdsprache - großer Wert gelegt.

\section{Erhebung zur Übersetzungsbewertung und Fehlerkorrektur}

\subsection{Erhebungssituation}

Um die Fragen, die in der Einleitung gestellt wurden, zu beantworten, habe ich in unserem Masterausbildungsprogramm für Übersetzen und Dolmetschen ein Seminar dem Thema „Übersetzungsfehler und Lektorieren“ gewidmet und den TeilnehmerInnen mehrere Aufgaben zum Selbstkorrigieren, Lektorieren sowie Bewerten gegeben. ${ }^{5}$ Die Aufgabentypen waren für die Studierenden neu, da studentisches Lektorieren und Bewerten in den Kursen - nicht nur bei uns, sondern in Übersetzungsstudiengängen generell - nicht oder nur selten geübt werden. Das ist deswegen bedauerlich, weil die Studierenden dadurch wichtige, im Berufsleben nötige Kompetenzen entwickeln könnten (vgl. Fischer 2011: 76).

Am erwähnten Seminar nahmen sieben Studierende teil: Sechs waren im 2. Semester, eine Studentin im 4. Semester. Als Textgrundlage für die Seminaraufgaben dienten die eigenen Übersetzungen der Studierenden aus dem Ungarischen ins Deutsche, die sie im Rahmen des Projekts „DULKO: Deutschungarisches Lernerkorpus“ am Lehrstuhl für Germanistische Linguistik der

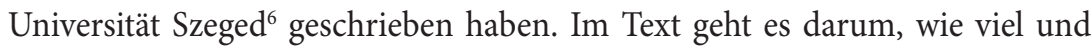
warum Frauen in ihren verschiedenen Lebensphasen weinen. Der Ausgangstext besteht aus ca. 200 Wörtern. Das Übersetzen erfolgte unter Aufsicht und es durften dabei keine Hilfsmittel benutzt werden.

Der Nutzen von solchen Aufgaben wird auch in der unterrichtsmethodologischen Fachliteratur thematisiert (z.B. Dróth 2017: 294, Fischer 2011: 76).

6 http://szegedigermanisztika.hu/index.php/de/forschung/projekte 
Im Folgenden werden die einzelnen Aufgaben zusammengefasst und die durch sie gewonnenen Daten präsentiert sowie ausgewertet.

\section{2 Übersetzungsbewertung aus der Perspektive von Studierenden}

Zur Bewertung bekamen die Studierenden drei Aufgaben: Nachdem sie die Möglichkeit gehabt hatten, ihre ursprüngliche Übersetzung zu Hause mit Hilfsmitteln zu korrigieren, mussten sie die Qualitätsstufe (s. Fußnote 1) beider Versionen einschätzen. Den ersten Text stuften alle als Rohübersetzung ein, in dem eben ohne Wörterbücher an einigen Stellen Lexeme und grammatische Informationen wie Genus oder Pluralformen fehlerhaft waren. Die korrigierte Übersetzung bewertete nur eine Person als druckreif, die anderen tendierten zur Informationsübersetzung, da sie hinsichtlich der stilistischen Perfektheit unsicher waren. Wie wir sehen werden, enthielten jedoch noch alle verbesserten Versionen sprachliche Fehler (s. 3.3), sodass sie nur als schlechtere Informationsübersetzungen oder sogar als Rohübersetzungen bezeichnet werden können.

Die zweite Aufgabe war, dass die TeilnehmerInnen die schon korrigierte Übersetzung eines Kommilitonen / einer Kommilitonin holistisch (und diagnostisch) kurz bewerten mussten. Die Bewertung besprachen die Betroffenen dann in Partnerarbeit. Damit übten sie auch, wie man Übersetzungen diplomatisch kritisieren und sich selbst verteidigen kann (vgl. Fischer 2011: 81). Ich selber habe zu jeder Übersetzung ebenfalls eine ähnliche Bewertung verfasst, sodass jede/r Studierende die zwei Beurteilungen vergleichen konnte. Im Nachhinein wurden sie darum gebeten, über die Aufgabe zu reflektieren, indem sie folgende Fragen beantworteten:

- Wie fanden Sie die Herausforderung, selber eine Bewertung abzugeben?

- $\quad$ Gab es größere Unterschiede zwischen der Beurteilung der Dozentin und der Ihres Peers?

- $\quad$ Sind Sie einverstanden mit den zwei Bewertungen?

- $\quad$ Geben die Bewertungen genug Rückmeldung, sind sie also nützlich?

Die Umfrage erbrachte, dass die meisten TeilnehmerInnen (5 von 7) die Aufgabe schwierig fanden. Als Grund nannten sie folgende Probleme: 
- $\quad$ Es war nicht einfach, eine kurze, nur das Wesentliche behandelnde Beurteilung zu schreiben (1 Person) bzw. ihre Gedanken richtig auszudrücken (1 Person).

- $\quad$ Es war noch schwieriger, im Text von anderen die Fehler zu bemerken als im eigenen (2 Personen).

- $\quad$ Problematisch fanden sie auch die Verwirklichung der Objektivität, da sie wussten, wessen Übersetzung sie bewerteten, und Angst hatten, wie diese Person reagieren wird (2 Personen).

- $\quad$ Sie waren unsicher, was als Fehler gilt (4 Personen). 3 Personen konnten z.B. nicht so richtig entscheiden, ob die Wortwahl oder eine Formulierung im Text fehlerhaft war, wenn sie selber ein anderes Wort oder andere Ausdrücke benutzt hätten. ${ }^{7}$

Die Aufgabenbewältigung erleichterte für sie auf der anderen Seite, dass

- $\quad$ sie keine detaillierte, auf Einzelheiten eingehende Bewertung abgeben mussten (1 Person),

- $\quad$ der Text nicht korrigiert und die einzelnen Fehler nicht markiert werden mussten (2 Personen).

Die jeweils zwei Bewertungen, die sie bekamen, stimmten größtenteils überein, vor allem was die früher im theoretischen Teil des Seminars behandelten Bewertungsaspekte betrifft. D.h. die vorkommenden Fehlertypen haben sie trotz ihrer Behauptung, einzelne Fehler nur mit Mühe identifizieren zu können, schon richtig genannt. Es gab nur wenige und kleinere Unterschiede, hauptsächlich weil ich bei 2 Leuten bestimmte Fehler kritischer einstufte als ihr Peer.

Im Allgemeinen akzeptierten sie die Bewertungen. Als Einwand wurde von 2 Personen erwähnt, dass sie ohne Fehlermarkierung und Erklärung nicht wissen, was und wo falsch übersetzt wurde, was für sie die Gültigkeit der Beurteilung einigermaßen relativiert. Eine weitere Person schrieb, dass sie die von ih-

Eine Studentin gab sogar an, dass sie sich in dieser Hinsicht bei der Beurteilung darauf stützte, dass die Verfasserin des Textes meistens gute Noten bekommt, daher müsse auch diese Übersetzung guter Qualität sein. 
rer Freundin angefertigte Beurteilung eben wegen ihrer Freundschaft erst dann ernst nehmen konnte, als sie meine ähnlich positive Bewertung gelesen hat.

Das führt schon zur letzten von mir gestellten Frage, ob die KursteilnehmerInnen die diagnostisch-holistische Rückmeldung nützlich fanden. Hier gehen die Meinungen auseinander, bzw. wiesen die Studierenden auf sowohl positive als auch negative Aspekte hin. Als Vorteil wurde empfunden, dass eine globale Bewertung

- $\quad$ durch die allgemeine Formulierung die evtl. größere Anzahl der Fehler verdeckt, man wird deshalb nicht mit vielen Korrekturen konfrontiert und eingeschüchtert (1 Person),

- $\quad$ helfen kann, konstruktive Kritik anzunehmen (1 Person),

- $\quad$ ermöglicht, über die einzelnen Bewertungsaspekte (Inhalt, Register usw.) getrennt die Meinung zu äußern und Rückmeldung zu bekommen (1 Person),

- $\quad$ die gute oder schlechte Gesamtqualität der Übersetzung besser hervorhebt (2 Personen),

- $\quad$ eine nützliche Rückmeldung über die eigene Leistung geben kann, vorausgesetzt sie ist objektiv (5 Personen).

Als nachteilig galt, dass diese Bewertung

- $\quad$ im Vergleich zur investierten Übersetzungsarbeit viel zu kurz ist (1 Person),

- $\quad$ eher bei kürzeren Übersetzungen genug Rückmeldung gibt (1 Person),

- $\quad$ subjektiv ausfallen kann (3 Personen),

- $\quad$ nicht eindeutig und detailliert genug ist, sodass man aus den Fehlern nicht lernen kann (4 Personen).

Als Bilanz kann man festhalten, dass die Studierenden die meisten Probleme damit hatten, die Fehler eindeutig zu identifizieren. Dazu gehört, dass sie sich bei dieser zweiten Aufgabe überraschenderweise kaum irgendwelcher Hilfsmittel, etwa Wörterbücher oder Grammatiken, bedienten. Das alles kann u.a. darauf zurückgeführt werden, dass sie im Unterricht kaum Lektorieren oder Selbstkorrektur üben. 
Neben der allgemeinen Auffassung, dass die holistische Bewertung nützlich sein kann und gegenüber der analytischen Methode mehrere Vorteile aufweist, behaupteten die Befragten, dass sie aus solchen Rückmeldungen kaum lernen können, was aber gerade der Sinn des Unterrichts wäre. Dazu kommt noch, dass sie sich - auch mündlich im Plenum - eher skeptisch über diese Methode äußerten in dem Sinne, dass sie davon überzeugt waren, wenn sie von DozentInnen nur eine kurze globale Begründung zur jeweiligen Note für ihre Übersetzung bekommen, sei das subjektiver und unzuverlässiger als eine detaillierte analytische Bewertung. Eine Studentin sagte z.B., dass sie von einer Lehrkraft schon eine holistische Beurteilung bekam, ohne davor eine analytische bekommen zu haben, weswegen sie nicht glaubte, dass ihre Übersetzung tatsächlich gut war. Sie konnte nämlich aufgrund der globalen Kritik nicht einschätzen, ob die jeweilige Lehrkraft den Text gründlich gelesen hat oder nicht. Ähnlich stellt auch Dróth (2011: 10-11) fest, dass Studierende meist eine detaillierte, analytische Bewertung wünschen - obwohl sie nicht unbedingt objektiver und zuverlässiger ist als eine holistische. Diese Problematik ist deswegen von Bedeutung, weil es auch für die Motivation der Studierenden wichtig wäre, dass sie die Bewertungen der DozentInnen akzeptieren und fair finden (Fischer 2011: 78).

\subsection{Studentische Fehlerkorrektur}

Um zu testen, wie erfolgreich die SeminarteilnehmerInnen Fehlersuche und korrektur bewältigen, mussten sie ihre eigene Übersetzung zuerst ohne, dann mit Hilfsmitteln verbessern. ${ }^{8}$ Ich habe sie darum gebeten, die identifizierten Fehler in einer Tabelle aufzulisten und zu korrigieren.

Ohne Hilfsmittel wurden Textelemente in folgender Anzahl korrigiert:

\begin{tabular}{|l|c|c|c|c|c|c|c|}
\hline \multicolumn{1}{|c|}{ Personen: } & $\mathbf{1}$ & $\mathbf{2}$ & $\mathbf{3}$ & $\mathbf{4}$ & $\mathbf{5}$ & $\mathbf{6}$ & $\mathbf{7}$ \\
\hline Gute Korrektur & 8 & 19 & 20 & 14 & 5 & 7 & 17 \\
\hline Schlechte Korrektur & 1 & 4 & 7 & - & - & 1 & - \\
\hline Unnötige Korrektur & 2 & 20 & 13 & 5 & 2 & 4 & 4 \\
\hline
\end{tabular}

Tab. 1: Ohne Hilfsmittel korrigierte Textelemente

\footnotetext{
8 So eine Aufgabe kann auch dazu beitragen, dass Studierende ihre Selbstbeurteilungskompetenz trainieren (Fischer 2011: 78).
} 
In den ca. 270 Wörter enthaltenden Übersetzungen änderten die Studierenden im Durchschnitt 20 Elemente. Am wenigsten korrigierte Person 5 (7 Elemente), am meisten Person 2 (43 Elemente). Aus Tabelle 1 geht auch hervor, dass die SeminarteilnehmerInnen ihre Fehler in den meisten Fällen gut korrigierten (z.B. in jeden Lebensalter $>$ in jedem Lebensalter). Falsche Korrekturen, wo also eine Textstelle für eine fehlerhafte Version ausgetauscht wurde, kamen eher vereinzelt vor (z.B. in ihrer ganzen Leben > in ihren ganzen Leben). Dagegen wurden relativ häufig (2-20mal) unnötige Änderungen vorgenommen (z.B. wöchentlich > jede Woche; Änderung von Satzgrenzen).

Die Studierenden mussten auch explizit nennen, was sie jeweils korrigiert haben. Sie operierten dabei mit den üblichen linguistischen Fehlerkategorien, die von den DozentInnen allgemein verwendet werden. Auf pragmatische und kulturelle Aspekte gingen sie nicht ein, da in der ursprünglichen Aufgabenstellung der Erhebung im Rahmen des DULKO-Projekts weder eine Zielgruppe noch eine Kommunikationssituation angegeben wurde sowie sich keine kulturellen Unterschiede hinsichtlich Textsortenkonventionen ergaben. Tabelle 2 zeigt, zu welchen linguistischen Bereichen die Änderungen angegeben wurden:

\begin{tabular}{|l|c|c|c|c|c|c|c|}
\hline \multicolumn{1}{|c|}{ Personen: } & $\mathbf{1}$ & $\mathbf{2}$ & $\mathbf{3}$ & $\mathbf{4}$ & $\mathbf{5}$ & $\mathbf{6}$ & $\mathbf{7}$ \\
\hline Interpunktion & - & - & 2 & 4 & - & - & - \\
\hline Rechtschreibung & 3 & 2 & 6 & 1 & - & 1 & 3 \\
\hline $\begin{array}{l}\text { Lexik/Semantik (Inhalt, } \\
\text { Umformulierung) }\end{array}$ & 2 & 30 & 11 & 5 & 5 & 8 & 6 \\
\hline Morphologie & 3 & 4 & 13 & 5 & 1 & 1 & 8 \\
\hline Syntax & 3 & 5 & 7 & 4 & 1 & 2 & 4 \\
\hline Textlinguistik & - & 1 & 1 & - & - & - & - \\
\hline
\end{tabular}

Tab. 2: Linguistische Bereiche der Änderungen ohne Hilfsmittel

In größter Anzahl wurden Korrekturen morphologischer und lexikalischer (z.T. inhaltlicher) Art durchgeführt (z.B. Bei größeren Kinder > Bei größeren Kindern; dieser Zahl > diese Zahl; psychischer Gründe > seelischer Verletzungen; eine Frau $>$ eine durchschnittliche Frau). Dann folgen nach der Häufigkeit orthografieund syntaxbezogene Änderungen, etwa bei der Wortstellung, Artikelsetzung oder Satzgrenzenbestimmung (z.B. Letzlich > Letztlich; dominieren die Ängste 
> dominieren Ängste; reagieren wir auf alle Geschehnisse natürlich empfindlich > reagieren wir natürlich auf alle Geschehnisse empfindlich). Textlinguistische und Interpunktionskorrekturen waren in der Erhebung selten zu finden (z.B. in $\underline{\text { den }}$ verschiedenen Lebensphasen > in ihren verschiedenen Lebensphasen; die Gründe $>$ diese Gründe; zum Beispiel Hunger > zum Beispiel: Hunger; was in späteren Lebensphasen, auch über 26 Jahren so bleibt > was in späteren Lebensphasen, auch über 26 Jahren, so bleibt).

In einer zweiten Runde hatten die Studierenden die Möglichkeit, ihre Übersetzung mit Hilfsmitteln zu verbessern. Wie man Tabelle 3 entnehmen kann, wurden in dieser Phase weniger Änderungen durchgeführt: im Durchschnitt 11, am wenigsten von Person 6 (3 Elemente), am meisten von Person 7 (22 Elemente).

\begin{tabular}{|l|c|c|c|c|c|c|c|}
\hline \multicolumn{1}{|c|}{ Personen: } & $\mathbf{1}$ & $\mathbf{2}$ & $\mathbf{3}$ & $\mathbf{4}$ & $\mathbf{5}$ & $\mathbf{6}$ & $\mathbf{7}$ \\
\hline Gute Korrektur & 14 & 8 & 2 & 7 & 3 & 2 & 17 \\
\hline Schlechte Korrektur & - & 1 & 1 & 2 & - & - & - \\
\hline Unnötige Korrektur & 4 & 5 & 2 & 2 & 4 & 1 & 5 \\
\hline
\end{tabular}

Tab. 3: Mit Hilfsmitteln korrigierte Textelemente

Auch hier überwiegt die gute Korrektur, also wenn fehlerhafte Textpassagen richtig geändert wurden (z.B. das Durchschnitt > der Durchschnitt; physische

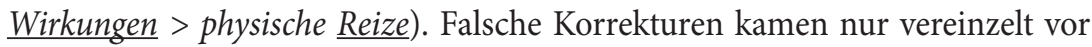
(z.B. überraschend > erschreckend; Welterfahrung > Erkennung der Welt statt Entdeckung der Welt). Die Studierenden führten aber auch wieder Änderungen durch, die unnötig waren (z.B. neulich > vor kurzer Zeit; Kleidungsstücke tragen > sich Kleidungsstücke anziehen).

\begin{tabular}{|l|c|c|c|c|c|c|c|}
\hline \multicolumn{1}{|c|}{ Personen: } & $\mathbf{1}$ & $\mathbf{2}$ & $\mathbf{3}$ & $\mathbf{4}$ & $\mathbf{5}$ & $\mathbf{6}$ & $\mathbf{7}$ \\
\hline Rechtschreibung & - & - & - & 1 & - & - & 2 \\
\hline $\begin{array}{l}\text { Lexik/Semantik (Inhalt, } \\
\text { Umformulierung) }\end{array}$ & 15 & 14 & 5 & 7 & 5 & 3 & 16 \\
\hline Morphologie & 3 & - & - & 1 & 1 & - & 4 \\
\hline Syntax & - & - & - & 2 & 1 & - & - \\
\hline
\end{tabular}

Tab. 4: Linguistische Bereiche der Änderungen mit Hilfsmitteln 
Anhand Tabelle 4 haben die Studierenden die Hilfsmittel (Wörterbücher, Online-Korpora) eindeutig vor allem dazu genutzt, um für bestimmte ungarische Wörter und Ausdrücke passende deutsche Entsprechungen zu suchen (z.B. die Gründe sind wechselnd > die Gründe sind vielfältig; zum ersten Blick > auf den ersten Blick; dieses ㅁata (sic!) > dieses Verhältnis; Forscher haben gesucht $>$ es wurde erforscht). Die Schreibweise von Wörtern (z.B. Emphatie > Empathie), Genus (starken Emphatie > starke Empathie; der Dauer > die Dauer) und Wortstellung (z.B. nämlich es wurde festgestellt > es wurde nämlich festgestellt) oder die Verwendung von Präpositionen (weint für 2 Stunden $>$ weint 2 Stunden) wurden viel weniger nachgeschlagen und korrigiert.

Die Studierenden haben leider Fehler verschiedener Art in folgender Anzahl übersehen:

\begin{tabular}{|l|c|c|c|c|c|c|c|}
\hline \multicolumn{1}{|c|}{ Personen: } & $\mathbf{1}$ & $\mathbf{2}$ & $\mathbf{3}$ & $\mathbf{4}$ & $\mathbf{5}$ & $\mathbf{6}$ & $\mathbf{7}$ \\
\hline Interpunktion & - & 1 & 3 & - & - & - & 1 \\
\hline Rechtschreibung & 1 & - & 1 & - & 1 & - & - \\
\hline $\begin{array}{l}\text { Lexik/Semantik (Inhalt, } \\
\text { Umformulierung) }\end{array}$ & 2 & - & 4 & 4 & 4 & - & 2 \\
\hline Morphologie & 3 & 8 & 9 & 3 & 5 & 4 & 10 \\
\hline Syntax & 6 & 3 & 2 & 2 & 2 & 4 & 2 \\
\hline Textlinguistik & - & - & - & - & - & 1 & - \\
\hline Stilistik, Register & - & - & 2 & - & - & - & 2 \\
\hline
\end{tabular}

Tab. 5: Übersehene, nicht korrigierte Fehler

In den überarbeiteten Texten kamen noch Fehler vor, im Durchschnitt 13 (Tiefstwert: 9, Höchstwert: 21). Die meisten Fehler waren vorwiegend grammatischer Art, also morphologische (z.B. die physische Wirkungen; 16 Monaten des Lebens) und syntaktische Fehler (vor allem Wortstellung oder Artikelverwendung, z.B. in ersten Phasen unseres Lebens). Danach folgt der Bereich Lexik/ Semantik (die Welt erkennen statt kennen lernen; Letztlich statt Vor kurzem). Weitere Fehler ergaben sich in Interpunktion (das 26_Lebensjahr), Rechtschreibung (Wochentag Morgen; der selbe), Textlinguistik (... Es zeigt uns eine andere Forschung, die ... statt Das zeigt uns ....) und Stilistik (wegen körperlichen und seelischen Erkrankungen) eher selten. 
Tabelle 6 zeigt das Gesamtbild der studentischen Korrekturen:

\begin{tabular}{|l|c|c|c|c|c|c|c|}
\hline \multicolumn{1}{|c|}{ Personen: } & $\mathbf{1}$ & $\mathbf{2}$ & $\mathbf{3}$ & $\mathbf{4}$ & $\mathbf{5}$ & $\mathbf{6}$ & $\mathbf{7}$ \\
\hline Gute Korrektur & 22 & 27 & 22 & 21 & 8 & 9 & 34 \\
\hline $\begin{array}{l}\text { Schlechte oder unter- } \\
\text { bliebene Korrektur }\end{array}$ & 13 & 17 & 29 & 11 & 12 & 10 & 17 \\
\hline Unnötige Korrektur & 6 & 25 & 15 & 7 & 6 & 5 & 9 \\
\hline
\end{tabular}

Tab. 6: Durchgeführte und unterbliebene Korrekturen insgesamt

Dieser Erhebungsteil hat zu folgenden Erkenntnissen geführt: Da die Studierenden im Lektorieren nicht trainiert waren, hatten sie tatsächlich allgemein Probleme mit der Identifizierung von Fehlern. Das manifestierte sich vor allem in der Zahl der unnötigen Änderungen, die sie in ihren Übersetzungen durchführten. Die hohe Zahl der übersehenen Fehler und der schlechten Korrekturen (wo also fehlerfreie Textstellen inkorrekt geändert wurden) deutet darauf hin, dass die Studierenden allgemein noch sprachliche Kompetenzmängel haben. Interessant ist gerade in dieser Hinsicht, dass sie sich trotzdem zugemutet haben, ihre Übersetzung größtenteils ohne Hilfsmittel zu korrigieren. Dagegen wurden am meisten lexikalisch-inhaltliche und morphologische Änderungen vorgenommen, bei denen man eigentlich einfach nachschauen und sichergehen kann.

\section{Fazit}

Anhand der Ergebnisse der ganzen Erhebung kann Folgendes festgehalten werden: Studierende halten Aufgaben für interessant und nützlich, wenn sie Übersetzungen selbst beurteilen und korrigieren müssen. Einerseits üben sie dadurch Kompetenzen ein, die sie im Berufsleben brauchen werden. Andererseits werden sie automatisch darauf aufmerksam, wie viele Fehler sie generell vor der Abgabe ihrer Texte schon mit einer letzten Überprüfungsrunde beheben könnten (vgl. dazu Fischer 2011: 78).

Die über die holistische Bewertung geäußerten Meinungen der Studierenden sollten wir Lehrenden meines Erachtens im Unterricht unbedingt be- 
rücksichtigen und diese Methode nur mit Vorsicht, z.B. in einigen gut geplanten Unterrichtssituationen oder mit analytischer Bewertung kombiniert, verwenden.

Eine weitere Erkenntnis und Empfehlung ist, dass man im Unterricht mehr Lektorieren und Selbstkorrektur üben sollte, um Kompetenzen wie Selbsteinschätzung, Fehlerfindung, Rationalisierung der Eingriffe im Text und Hilfsmittelbenutzung beim Lektorieren zu entwickeln.

\section{Literatur}

Dróth, Júlia (2011): A fordítások értékelése a szakfordítóképzésben és a fordítói munka világában [Übersetzungsbewertung in der Fachübersetzerausbildung und in der Arbeitspraxis]. In: Fordítástudomány XIII/2, 5-36.

Dróth, Júlia (2017): Kutatási kérdések a fordítások értékelése témakörben [Forschungsfragen zum Thema Übersetzungsbewertung]. In: Kóbor, Márta / Csikai, Zsuzsanna (Hrsg.): Iránytü az egyetemi fordítóképzéshez. A kompetenciafejlesztés új fókuszai. Pécs: Kontraszt, 289-302.

Fischer, Márta (2011): A társas és önértékelés szerepe a fordítás oktatásában [Die Rolle der Selbst- und Peerbewertung im Übersetzungsunterricht]. In: Dróth, Júlia (Hrsg.): Szaknyelv és Szakfordítás. Tanulmányok a szakfordítás és a fordítóképzés aktuális témáiról 2010-2011. Gödöllö: Szent István Egyetem GTK, 76-82.

Hönig, Hans G. (1997): Positions, Power and Practice: Functionalist Approaches and Translation Quality Assessment. In: Current Issues in Language \& Society. Bd. 4/1. 6-34.

Klaudy, Kinga (2007): A fordítási hibák értékelése az életben, a képzésben és a vizsgán [Die Bewertung von Übersetzungsfehlern in der Arbeitspraxis, in der Ausbildung und in Prüfungen]. In: Klaudy, Kinga: Nyelv és fordítás. Válogatott fordítástudományi tanulmányok. Budapest: Tinta Könyvkiadó (Segédkönyvek a nyelvészet tanulmányozásához 68), 253-260.

Koller, Werner (2011): Einführung in die Übersetzungswissenschaft. 8., neub. Aufl. Tübingen / Basel: Francke.

Kußmaul, Paul (2015): Verstehen und Übersetzen. Ein Lehr- und Arbeitsbuch. 3., überarb. und erw. Aufl. Tübingen: Narr. 
$\mathrm{MQM}=$ Deutsches Forschungszentrum für Künstliche Intelligenz $\mathrm{GmbH}$ / German Research Center for Artificial Intelligence (DFKI) and QTLaunchPad (2015): Multidimensional Quality Metrics (MQM) Definition: http:// www.qt21.eu/mqm-definition/definition-2015-12-30.html (gesichtet am 06.12.2018).

Nord, Christiane (2010): Fertigkeit Übersetzen. Ein Kurs zum Übersetzenlehren und -lernen. Berlin: BDÜ Fachverlag.

Schäffner, Christina (2004): Systematische Übersetzungsdefinitionen. In: Frank, Armin P. / Greiner, Norbert / Hermans, Theo / Kittel, Harald / Koller, Werner / Lambert, Jose / Paul, Fritz (Hrsg.): Übersetzung. Ein internationales Handbuch zur Übersetzungsforschung. Berlin / New York: de Gruyter (HSK 26.1), 101-117.

Stolze, Radegundis (2013): Fachübersetzen - Ein Lehrbuch für Theorie und Praxis. 3., korr. Aufl. Berlin: Frank \& Timme. 